\title{
Radiation of Quantized Black Hole
}

\author{
I.B. Khriplovich ${ }^{1}$ \\ Budker Institute of Nuclear Physics \\ 630090 Novosibirsk, Russia, \\ and Novosibirsk University
}

\begin{abstract}
The maximum entropy of a quantized surface is demonstrated to be proportional to the surface area in the classical limit. The general structure of the horizon spectrum and the value of the Barbero-Immirzi parameter are found. The discrete spectrum of thermal radiation of a black hole fits naturally the Wien profile. The natural widths of the lines are very small as compared to the distances between them. The total intensity of the thermal radiation is calculated.
\end{abstract}

\footnotetext{
${ }^{1}$ khriplovich@inp.nsk.su
} 


\section{Introduction}

The idea of quantizing the horizon area of black holes was put forward many years ago by Bekenstein in the pioneering article [1]. He pointed out that reversible transformations of the horizon area of a nonextremal black hole found by Christodoulou and Ruffini [2, 3] have an adiabatic nature. Of course, the quantization of an adiabatic invariant is perfectly natural, in accordance with the correspondence principle.

Once this hypothesis is accepted, the general structure of the quantization condition for large quantum numbers gets obvious, up to an overall numerical constant $\beta$. The quantization condition for the horizon area $A$ should be

$$
A=\beta l_{p}^{2} N
$$

where $N$ is some large quantum number 4]. Indeed, the presence of the Planck length squared $l_{p}^{2}=k \hbar / c^{3}$ is only natural in this quantization rule. Then, for the horizon area $A$ to be finite in the classical limit, the power of $N$ should be the same as that of $\hbar$ in $l_{p}^{2}$. This argument can be checked by considering any expectation value in quantum mechanics, nonvanishing in the classical limit. It is worth mentioning that there are no compelling reasons to believe that $N$ is an integer. Neither there are compelling reasons to believe that the spectrum (11) is equidistant [5, 6].

On the other hand, formula (II) can be interpreted as follows. The whole horizon area $A$ is split into elements of typical size $\sim l_{p}^{2}$, each of them giving a contribution to the large quantum number $N$. This scheme arises in particular in the framework of loop quantum gravity (LQG) [7-11].

A quantized surface in LQG looks as follows. One ascribes to it a set of edges. Each edge is supplied with an integer or half-integer "angular momentum" $j$ :

$$
j=1 / 2,1,3 / 2, \ldots .
$$

The projections $m$ of these "angular momenta" run as usual from $-j$ to $j$. The area of a surface is

$$
A=8 \pi \gamma l_{p}^{2} \sum_{i} \sqrt{j_{i}\left(j_{i}+1\right)} .
$$

The numerical factor $\gamma$ in (3) cannot be determined without an additional physical input. This free (so-called Barbero-Immirzi) parameter [12, 13] corresponds to a family of inequivalent quantum theories, all of them being viable without such an input.

We mention that though the spectrum (3) is not equidistant, it is not far away from it. Indeed, even for the smallest quantum number $j=1 / 2, \sqrt{j(j+1)}$ can be approximated by

$j+1 / 2$ with an accuracy $13 \%$. And the approximation $\sqrt{j(j+1)} \approx j+1 / 2$ gets better and better with growing $j$, i.e. the spectrum (3) approaches an equidistant one more and more. This feature of the spectrum (3) is of interest in connection with the observation due to Bekenstein: quantum effects result in the following lower bound on the change of the horizon area $\Delta A$ under an adiabatic process:

$$
(\Delta A)_{\min }=\xi l_{p}^{2}
$$


here $\xi$ is a numerical factor reflecting "the inherent fuzziness of the uncertainty relation" 14. Of course, right-hand-side of formula (4) is proportional to $\hbar$, together with the Planck length squared $l_{p}^{2}$.

Due to the uncertainty of the numerical factor $\xi$ itself, one cannot see any reason why $\xi$ should not slightly change from one act of capture to another. So, the discussed quasiequidistant spectrum (3) agrees with the bound (4), practically as well as the equidistant one. Below we will come back to relation (4).

As to the unknown parameter $\gamma$ in (3), the first attempts to fix its value, based on the analysis of the black hole entropy, were made in [15, 16. However, these attempts did not lead to concrete quantitative results.

Then it was argued in [17] that for the black hole horizon all quantum numbers $j$ are equal to $1 / 2$ (as it is the case in the so-called "it from bit" model formulated earlier by Wheeler [18]). With these quantum numbers, one arrives easily at the equidistant area spectrum and at the value $\gamma=\ln 2 /(\pi \sqrt{3})$ for the Barbero-Immirzi parameter. However, the result of [17] was demonstrated in [5] to be certainly incorrect ${ }^{2}$ since it violates the so-called holographic bound formulated in [22-24]. According to this bound, among the spherical surfaces of a given area, it is the surface of a black hole horizon that has the largest entropy.

\section{Microcanonical Entropy of Black Hole}

On the other hand, this requirement of maximum entropy allows one to find the correct structure of the horizon area [25], which in particular is of crucial importance for the problem of radiation of a quantized black hole.

We consider in fact the "microcanonical" entropy $S$ of a quantized surface defined as the logarithm of the number of states of this surface for a fixed area $A$ (instead of fixed energy in common problems). Obviously, this number of states $K$ depends on the assumptions concerning the distinguishability of the edges.

To analyze the problem, it is convenient to rewrite formula (3) as follows:

$$
A=8 \pi \gamma l_{p}^{2} \sum_{j m} \sqrt{j(j+1)} \nu_{j m} .
$$

Here $\nu_{j m}$ is the number of edges with given $j$ and $m$. It can be demonstrated [5, 6] that the only reasonable assumption on the distinguishability of edges that may result in acceptable physical predictions (i.e. may comply both with the Bekenstein-Hawking relation and with the holographic bound) is as follows:

$$
\begin{array}{cccr}
\text { nonequal } j, & \text { any } m & \longrightarrow & \text { distinguishable; } \\
\text { equal } j, & \text { nonequal } m & \longrightarrow & \text { distinguishable; } \\
\text { equal } j, & \text { equal } m & \longrightarrow & \text { indistinguishable. }
\end{array}
$$

\footnotetext{
${ }^{2}$ Later, the result of [17] was criticized as well in [19, 20]. Then an error made in [17] was acknowledged [21]. We will demonstrate below that the result of [19, 20] is also incorrect.
} 
Under this assumption, the number of states of the horizon surface for a given number $\nu_{j m}$ of edges with momenta $j$ and their projections $j_{z}=m$, is obviously

$$
K=\nu ! \prod_{j m} \frac{1}{\nu_{j m} !}, \quad \text { where } \quad \nu=\sum_{j} \nu_{j}, \quad \nu_{j}=\sum_{m} \nu_{j m},
$$

and the corresponding entropy equals

$$
S=\ln K=\ln (\nu !)-\sum_{j m} \ln \left(\nu_{j m} !\right)
$$

The structures of the last expression and of formula (5) are so different that in a general case the entropy certainly cannot be proportional to the area. However, this is the case for the maximum entropy in the classical limit.

In this limit, with all effective "occupation numbers" large, $\nu_{j m} \gg 1$, we use the Stirling approximation so that the entropy is

$$
S=\nu \ln \nu-\sum_{j m} \nu_{j m} \ln \nu_{j m}
$$

We calculate its maximum for a fixed area $A$, i.e. for a fixed sum

$$
N=\sum_{j m}^{\infty} \sqrt{j(j+1)} \nu_{j m}=\text { const } .
$$

The problem reduces to the solution of the system of equations

$$
\ln \nu-\ln \nu_{j m}=\mu \sqrt{j(j+1)},
$$

where $\mu$ is the Lagrange multiplier for the constraining relation (9). These equations can be rewritten as

$$
\nu_{j m}=\nu e^{-\mu \sqrt{j(j+1)}},
$$

or

$$
\nu_{j}=(2 j+1) e^{-\mu \sqrt{j(j+1)}} \nu
$$

Now we sum expressions (12) over $j$, and with $\sum_{j} \nu_{j}=\nu$ arrive at the equation for $\mu$ :

$$
\sum_{j=1 / 2}^{\infty}(2 j+1) e^{-\mu \sqrt{j(j+1)}}=1
$$

Its solution is

$$
\mu=1.722 \text {. }
$$

Strictly speaking, the summation in formula (14) extends not to infinity, but to some $j_{\max }$. Its value follows from the obvious condition: none of $\nu_{j m}$ should be less than unity. Then, for $\nu \gg 1$ equation (11) gives

$$
j_{\max }=\frac{\ln \nu}{\mu}
$$


It is well-known that the Stirling approximation for $n$ ! has reasonably good numerical accuracy even for $n=1$. Due to it, formula (15) for $j_{\max }$ is not just an estimate, but has reasonably good numerical accuracy. The relative magnitude of the corresponding correction to (14) can be easily estimated as $\sim \ln \nu / \nu$.

Let us come back now to equation (10). When multiplying it by $\nu_{j m}$ and summing over $j m$, we arrive with the constraint (9) at the following result for the maximum entropy for a given value of $N$ :

$$
S_{\max }=1.722 \mathrm{~N},
$$

so that with the Bekenstein-Hawking relation and formula (15) we find the value of the Barbero-Immirzi parameter:

$$
\gamma=0.274
$$

Quite recently this calculation with the same result, though with somewhat different motivation, was reproduced in [26].

It should be emphasized that the above calculation is not special for LQG only, but applies (with obvious modifications) to a more general class of approaches to the quantization of surfaces. The following assumption is really necessary here: the surface should consist of sites of different sorts, so that there are $\nu_{i}$ sites of each sort $i$, with a generalized effective quantum number $r_{i}$ (here $\sqrt{j(j+1)}$ ), and a statistical weight $g_{i}$ (here $2 j+1$ ). Then in the classical limit, with given functions $r_{i}$ and $g_{i}$ the maximum entropy of a surface can be found, at least numerically, and it is certainly proportional to the area of the surface.

As to previous attempts to calculate $\gamma$, one should indicate an apparent error in state counting made in [19, 20]. It can be easily checked that the transition from formula (25) to formulae (29), (36) of [19] performed therein and then employed in [20], is certainly valid under the assumption that for each quantum number $j$ only two maximum projections $\pm j$ are allowed. But therefore it cannot hold for the correct number $2 j+1$ of the projections. No wonder that the equation for the BI parameter in [20] looks as

$$
2 \sum_{j=1 / 2}^{\infty} e^{-\mu \sqrt{j(j+1)}}=1
$$

instead of ours (13) (see also the discussion of (18) in [26]).

The conclusion is obvious. Any restriction on the number of admissible states for the horizon, as compared to a generic quantized surface, be it the restriction to

$$
j=1 / 2, \quad m= \pm 1 / 2
$$

made in [17, or the restriction to

$$
\text { any } j, \quad m= \pm j,
$$

made in 19, 20], results in a conflict with the holographic bound. 


\section{Quantization of Rotating Black Hole}

When discussing the radiation spectrum of quantized black holes, one should take into account the selection rules for angular momentum. Obviously, radiation of any particle with nonvanishing spin is impossible if both initial and final states of a black hole are spherically symmetric. Therefore, to find the radiation spectrum, the quantization rule for the mass of a Schwarzschild black hole should be generalized to that of a rotating Kerr black hole.

To derive the quantization rule for Kerr black hole, we come back to the thought experiment analyzed in 2, 3. Therein, under adiabatic capture of a particle with an angular momentum $j$, the angular momentum $J$ of a rotating black hole changes by a finite amount $j$, but the horizon area $A$ does not change. Of course, under some other variation of parameters it is the angular momentum $J$ that remains constant. In other words, we have here two independent adiabatic invariants, $A$ and $J$, for a Kerr black hole with a mass $M$.

Such a situation is quite common in ordinary mechanics. For instance, the energy of a particle with mass $m$ bound in the Coulomb field $U(r)=-\alpha / r$ is

$$
E=-\frac{m \alpha^{2}}{2\left(I_{r}+I_{\phi}\right)^{2}},
$$

where $I_{r}$ and $I_{\phi}$ are adiabatic invariants for the radial and angular degree of freedom, respectively. Of course, the energy $E$ is in a sense an adiabatic invariant also, but it is invariant only with respect to those variations of parameters under which both $I_{r}$ and $I_{\phi}$ remain constant. As to quantum mechanics, in it formula (19) goes into

$$
E=-\frac{m \alpha^{2}}{2 \hbar^{2}\left(n_{r}+1+l\right)^{2}},
$$

where $n_{r}$ and $l$ are the radial and orbital quantum numbers, respectively.

This example prompts the solution of the quantization problem for a Kerr black hole. It is conveniently formulated in terms of the so-called irreducible mass $M_{i r}$ of a black hole, related by definition to its horizon radius $r_{h}$ and area $A$ as follows:

$$
r_{h}=2 k M_{i r}, \quad A=16 \pi k^{2} M_{i r}^{2}
$$

Together with the horizon area $A$, the irreducible mass is an adiabatic invariant. In accordance with (3) and (9), it is quantized as follows:

$$
M_{i r}^{2}=\frac{1}{2} m_{p}^{2} N
$$

where $m_{p}^{2}=\hbar c / k$ is the Planck mass squared.

Of course, for a Schwarzschild black hole $M_{i r}$ coincides with its ordinary mass $M$. However, for a Kerr black hole the situation is more interesting. Here

$$
M^{2}=M_{i r}^{2}+\frac{J^{2}}{r_{h}^{2}}=M_{i r}^{2}+\frac{J^{2}}{4 k^{2} M_{i r}^{2}},
$$


where $J$ is the internal angular momentum of a rotating black hole.

Now, with the account for equation (22), we arrive at the following quantization rule for the mass squared $M^{2}$ of a rotating black hole:

$$
M^{2}=\frac{1}{2} m_{p}^{2}\left[\gamma N+\frac{J(J+1)}{\gamma N}\right] .
$$

Obviously, as long as a black hole is far away from an extremal one, i.e. while $\gamma N \gg J$, one can neglect the dependence of $M^{2}$ on $J$, and the angular momentum selection rules have practically no influence on the radiation spectrum of a black hole.

As to the mass and irreducible mass of a charged black hole, they are related as follows:

$$
M=M_{i r}+\frac{q^{2}}{2 r_{h}} ;
$$

here $q$ is the charge of the black hole. This formula has a simple physical interpretation: the total mass (or total energy) $M$ of a charged black hole consists of its irreducible mass $M_{i r}$ and of the energy $q^{2} / 2 r_{h}$ of its electric field in the outer space $r>r_{h}$.

With $r_{h}=2 k M_{i r}$, relation (25) can be rewritten as

$$
M^{2}=M_{i r}^{2}+\frac{q^{4}}{16 k^{2} M_{i r}^{2}}+\frac{q^{2}}{2 k} .
$$

Thus, for a charged black hole $M^{2}$ is quantized as follows:

$$
M^{2}=\frac{1}{2} m_{p}^{2}\left[\gamma N+\frac{q^{4}}{4 \gamma N}+q^{2}\right] .
$$

In fact, relations of this type (even in a more general form, for Kerr-Newman black holes, both charged and rotating) were presented already in the pioneering article [1, though with the equidistant quantization rule for $M_{i r}^{2}$, i.e. for the horizon area (see also [14]). More recently, the conclusion that the mass of a quantized black hole should be expressed via its quantized area and angular momentum, was made in the approach based on the notion of so-called isolated horizons [27, 28].

I do not mention here those attempts to quantize rotating and charged black holes which resulted in weird quantization rules for $\hat{J}^{2}$ and $e^{2} / \hbar c$.

\section{Radiation Spectrum of Quantized Black Hole}

It follows from expression (24) that for a rotating black hole the radiation frequency $\omega$, which coincides with the loss $\Delta M$ of the black hole mass, is

$$
\omega=\Delta M=T \mu \Delta N+\frac{1}{4 k M} \frac{2 J+1}{\gamma N} \Delta J
$$


where $\Delta N$ and $\Delta J$ are the losses of the area quantum number $N$ and of the angular momentum $J$, respectively. We have used here, in line with (24), the following identity for the Hawking temperature $T$ :

$$
T=\frac{\partial M}{\partial S}=\frac{1}{8 \pi k M} \frac{\partial M^{2}}{\partial M_{i r}^{2}}
$$

as well as formula (23).

In the same way, for a charged black hole one obtains with formula (27) the radiation frequency

$$
\omega=\Delta M=T \mu \Delta N+\frac{1}{4 k M}\left(2+\frac{q^{2}}{\gamma N}\right) q \Delta q,
$$

where $\Delta q$ is the loss of the charge.

We will be interested mainly in the first, temperature terms in (28) and (30), dominating everywhere but the vicinity of the extremal regime, where $J \rightarrow \gamma N$, or $q^{2} \rightarrow 2 \gamma N$, and $T \rightarrow 0$. The natural assumption is that the temperature radiation occurs when an edge with a given value of $j$ disappears, which means that

$$
\Delta N_{j}=r_{j}, \quad \omega_{j}=T \mu r_{j}
$$

Thus we arrive at the discrete spectrum with a finite number of lines. Their frequencies start at $\omega_{\min }=T \mu \sqrt{3} / 2$ and terminate at $\omega_{\max }=T \ln \nu$. We recall here that $j \leq j_{\max }=\ln \nu / \mu$, so that the number of lines is not so large, $\sim 10^{2}$, if the mass of black hole is comparable to that of the Sun. However, due to the exponential decrease of the radiation intensity with $\omega$ or $j$ (see below), the existence of $\omega_{\max }$ and finite number of lines are not of much importance.

To substantiate the made assumption, we come back to the lower bound (4) on the change of the horizon area under an adiabatic capture of a particle. The presence of the gap (4) in this process means that this threshold capture effectively consists in the increase by unity of the occupation number $\nu_{j m}$ with the smallest $j$, equal to $1 / 2$. If the capture were accompanied by a reshuffle of few occupation numbers, the change of the area could be easily made as small as one wishes. For instance, one could delete two edges with quantum numbers $j_{1}$ and $j_{2}$, and add an edge with a quantum number $j_{1}+j_{2}$. Obviously, with $j_{1,2} \gg 1$ the area increase could be made arbitrarily small.

It is only natural to assume that in the radiation process as well, changing few occupation numbers instead of one is at least strongly suppressed. In this way we arrive at equations (31).

Our next assumption, at least as natural as this one, is that the probability of radiation of a quantum with frequency $\omega_{j}$ is proportional to the occupation number $\nu_{j}$. Correspondingly, the radiation intensity $I_{j}$ at this frequency $\omega_{j}$ is proportional to $\nu_{j} \omega_{j}$ :

$$
I_{j} \sim \nu_{j} \omega_{j} \sim \nu(2 j+1) \omega_{j} \exp \left(-\omega_{j} / T\right)
$$

Let us compare this expression with the intensity of the black-body radiation in the Wien $\operatorname{limit} \omega / T \gg 1$,

$$
I(\omega)=A \frac{\omega^{3}}{4 \pi^{2}} \exp (-\omega / T) d \omega
$$


where $A$ is the area of a spherical black body. First of all, our relation (32) for $I_{j}$ reproduces directly the exponential factor of the Wien spectrum. Then, $d \omega$ in (33) goes over into $1 / 2 \mu T$ since the limit $\omega / T \gg 1$ corresponds in our problem to $\sqrt{j(j+1)} \gg 1$, i.e. to $\sqrt{j(j+1)} \simeq j+1 / 2$, and the minimum increment of $j$ is $1 / 2$. Now, to reproduce the Wien profile, we supplement relation (32) with the following factors: some "oscillator strength" proportional to $\omega_{j}$, obvious powers of $\mu T$, the Newton constant $k$ (necessary to transform $\nu$ into $A$ ), and obvious numerical ones. Thus we arrive at the final formula for the discrete radiation spectrum of a black hole:

$$
I_{j}=A T^{4} \frac{\mu^{4}}{8 \pi^{2}} j(j+1 / 2)(j+1) \exp (-\mu \sqrt{j(j+1)}) .
$$

Of course, since the Wien spectrum (33) corresponds to $j \gg 1$, one cannot guarantee the exact structure of $j$-dependence in formula (34), especially in the preexponential factor. For instance, it would be perhaps as legitimate to write therein $j^{3 / 2}(j+1)^{3 / 2}$ instead of $j(j+1 / 2)(j+1)$. However, this ambiguity is not as essential, at least numerically.

We note that since the black hole temperature $T$ is less than the minimum allowed frequency $\omega_{\text {min }}$, this spectrum has no Rayleigh-Jeans region at all.

Now, the emission probability for a quantum of frequency $\omega_{j}=T \mu r_{j}$, i.e. the width of the corresponding line, is

$$
\Gamma_{j}=\frac{I_{j}}{\omega_{j}}=A T^{3} \frac{\mu^{3}}{8 \pi^{2}}(j+1 / 2) \sqrt{j(j+1)} \exp (-\mu \sqrt{j(j+1)}) .
$$

The ratio of this natural line width to the distance $\Delta \omega_{j}=\omega_{j+1}-\omega_{j} \simeq 1 / 2 \mu T$ between the lines is very small numerically:

$$
\frac{\Gamma_{j}}{\Delta \omega_{j}} \simeq \frac{\mu^{2}}{16 \pi^{3}}(j+1 / 2)\left(\sqrt{j(j+1)} \exp (-\mu \sqrt{j(j+1)}) \lesssim 10^{-3} .\right.
$$

Thus, the radiation spectrum of an isolated black hole is really discrete.

At last, the total radiation intensity of a black hole is

$$
I=\sum_{j} I_{j}=0.150 A T^{4}
$$

The numerical coefficient in this expression is close to that in the total intensity of the common thermal radiation, i.e. to the Stefan-Boltzmann constant which is $\pi^{2} / 60=0.164$. The point is that the Rayleigh-Jeans contribution to the total intensity, which is completely absent in the present spectrum, would be small anyway.

Formulae (34) and (37) describe the thermal radiation not only of bosons, photons and gravitons. They describe as well the thermal radiation of fermions, massless neutrinos. However, in the last case a proper account for the number of the polarization states is necessary: for a two-component Dirac neutrino the numerical factors in formulae (34) and (37) will be two times smaller. 
In fact, it was argued long ago 29] that the discrete thermal radiation spectrum of a black hole, with the equidistant quantization rule for the horizon area, should fit the Wien profile.

On the other hand, our conclusion of the discrete radiation spectrum of a black hole in LQG differs drastically from that of [30] according to which the black hole spectrum in LQG is dense.

As to the nonthermal radiation of extremal black holes, described by the terms with $\Delta J$ and $\Delta q$ in formulae (28) and (30), these effects are due to tunneling (see relatively recent discussion of the subject, as well as detailed list of relevant references, in [31, 32]). The loss of a charge by a charged black hole is caused in fact by the Coulomb repulsion between the black hole and emitted particles with the same sign of charge. For a rotating black hole the reason is the interaction of angular momenta: particles (massless mainly), whose total angular momentum is parallel to that of a black hole, are repelled from it.

\section{Acknowledgements}

I appreciate numerous useful discussions with O.P. Sushkov. I am grateful also to J. Bekenstein for the correspondence; in particular, he has attracted my attention to the limit (4). An essential part of this work was done during my visit to School of Physics, University of New South Wales, Sydney; I wish to thank UNSW for the kind hospitality. The investigation was supported in part by the Russian Foundation for Basic Research through Grant No. 03-02-17612.

\section{References}

[1] J.D. Bekenstein, Lett. Nuovo Cimento, 11, 467 (1974).

[2] D. Christodoulu, Phys. Rev. Lett., 25, 1596 (1970).

[3] D. Christodoulu, R. Ruffini, Phys. Rev., D4, 3552 (1971).

[4] I.B. Khriplovich, Phys. Lett., B431, 19 (1998); preprint gr-qc/9804004 (1998).

[5] I.B. Khriplovich, Zh. Eksp. Teor. Fiz., 126, 527 (2004)

[Sov. Phys. JETP, 99, 460 (2004)]; preprint gr-qc/0404083 (2004).

[6] I.B. Khriplovich, preprint gr-qc/0409031 (2004).

[7] C. Rovelli, L. Smolin, Nucl. Phys., B442, 593 (1995); erratum, ibid. B456, 753 (1995); preprint gr-qc/9411005 (1994).

[8] A. Ashtekar, J. Lewandowski, Class. Quantum Grav., 14, 55 (1997); preprint gr-qc/9602046 (1996).

[9] R. Loll, Phys. Rev. Lett.,75, 3048 (1995); preprint gr-qc/9506014 (1995);

R. Loll, Nucl. Phys., B460, 143 (1996); preprint gr-qc/9511030 (1995). 
[10] R. De Pietri, C. Rovelli, Phys. Rev., D54, 2664 (1996);

preprint gr-qc/9602023 (1996).

[11] S. Frittelli, L. Lehner, C. Rovelli, Class. Quantum Grav. 13, 2921 (1996);

preprint gr-qc/9608043 (1996).

[12] G. Immirzi, Class. Quantum Grav., 14, L177 (1997); preprint gr-qc/9701052 (1997).

[13] C. Rovelli, T. Thiemann, Phys. Rev., D57, 1009 (1998);

preprint gr-qc/970559 (1997).

[14] J.D. Bekenstein, in Cosmology and Gravitation, ed. by M. Novello (Atlantisciences, France 2000) 1; gr-qc/9808028 (1998).

[15] C. Rovelli, Phys. Rev. Lett., 77, 3288 (1996); preprint gr-qc/9603063 (1996).

[16] K.V. Krasnov, Phys. Rev., D55, 3505 (1997); preprint gr-qc/9603025;

K.V. Krasnov, Gen. Rel. Grav., 30, 53 (1998); preprint gr-qc/9605047 (1996);

K.V. Krasnov, Class. Quantum Grav., 16, 563 (1999); preprint gr-qc/9710006 (1997).

[17] A. Ashtekar, J. Baez, A. Corichi, K. Krasnov, Phys. Rev. Lett., 80, 904 (1998); preprint gr-qc/9710007 (1997).

[18] J.A. Wheeler, in Sakharov Memorial Lectures in Physics, Moscow, vol. 2, p. 751 (1991).

[19] J. Lewandowski, M. Domagala, Class. Quantum Grav. 21, 5245 (2004); preprint gr-qc/0407051 (2004).

[20] K. Meissner, Class. Quantum Grav. 21, 5245 (2004); preprint gr-qc/0407052 (2004).

[21] A. Ashtekar, preprint gr-qc/0410054 (2004).

[22] J.D. Bekenstein, Phys. Rev., D23, 287 (1981).

[23] G. 't Hooft, in Salam Festschrift, Singapore (1993); preprint gr-qc/9310026 (1993).

[24] L. Susskind, J. Math. Phys., 36, 6377 (1995); preprint gr-qc/9710007 (1997).

[25] R.V. Korkin, I.B. Khriplovich, Zh. Eksp. Teor. Fiz., 122, 1 (2002)

[Sov. Phys. JETP, 95, 1 (2002) ]; preprint gr-qc/0112074 (2001).

[26] A. Ghosh, P. Mitra, preprint gr-qc//0411035 (2004).

[27] A. Ashtekar, S. Fairhurst, B. Krishnan, Phys. Rev., D62, 104025 (2000); preprint gr-qc/0005083 (2000).

[28] A. Ashtekar, C. Beetle, J. Lewandowski, Class. Quantum Grav., 19, 1195 (2002); preprint gr-qc/0111067 (2001). 
[29] J.D. Bekenstein, V.F. Mukhanov, Phys. Lett., B360, 7 (1995); preprint gr-qc/9505012 (1995).

[30] M. Barreira, M. Carfora, C. Rovelli, Gen. Rel. Grav., 28, 1293 (1996); preprint gr-qc/9603064 (1996).

[31] I.B. Khriplovich, Zh. Eksp. Teor. Fiz., 115, 1539 (1999)

[ Sov. Phys. JETP, 88, 845 (1999)]; preprint gr-qc/9812060 (1998).

[32] R.V. Korkin, I.B. Khriplovich, Zh. Eksp. Teor. Fiz., 121, 531 (2002) [Sov. Phys. JETP, 94, 453 (2002)]; preprint gr-qc/0107101 (2001). 